\title{
A Survey of Code-switching: Linguistic and Social Perspectives for Language Technologies
}

\author{
A. Seza Doğruöz \\ Ghent University, Gent, Belgium \\ as.dogruoz@ugent.be \\ Barbara E. Bullock \\ UT at Austin, Austin, USA \\ bbullockeaustin.utexas. edu
}

\author{
Sunayana Sitaram \\ Microsoft Research India, Bangalore, India \\ sunayana. sitaram@microsoft.com
}

\author{
Almeida Jacqueline Toribio \\ UT at Austin, Austin, USA \\ toribiodaustin.utexas.edu
}

\begin{abstract}
The analysis of data in which multiple languages are represented has gained popularity among computational linguists in recent years. So far, much of this research focuses mainly on the improvement of computational methods and largely ignores linguistic and social aspects of $\mathrm{C}-\mathrm{S}$ discussed across a wide range of languages within the long-established literature in linguistics. To fill this gap, we offer a survey of code-switching (C-S) covering the literature in linguistics with a reflection on the key issues in language technologies. From the linguistic perspective, we provide an overview of structural and functional patterns of C-S focusing on the literature from European and Indian contexts as highly multilingual areas. From the language technologies perspective, we discuss how massive language models fail to represent diverse C-S types due to lack of appropriate training data, lack of robust evaluation benchmarks for C-S (across multilingual situations and types of C-S) and lack of end-toend systems that cover sociolinguistic aspects of C-S as well. Our survey will be a step towards an outcome of mutual benefit for computational scientists and linguists with a shared interest in multilingualism and C-S.
\end{abstract}

\section{Introduction}

It is common for individuals in multilingual communities to switch between languages in various ways, in speech and in writing. In example 1, a bilingual child alternates between German and Turkish (in bold) to describe her teacher at school. Note that the Turkish possessive case marker (-si) is attached to a German noun (Karakoç and Herkenrath, 2019).

1. Frau Kummer. Echte Name-si Christa. Ms. Kummer. Real Name-Poss.3sg Christa. 'Ms. Kummer. (Her) real name is Christa'
The goal of this paper is to inform researchers in computational linguistics (CL) and language technologies about the linguistic and social aspects of code-switching (C-S) found in multilingual contexts (e.g. Europe and India) and how linguists describe and model them. Our intent is to increase clarity and depth in computational investigations of $\mathrm{C}-\mathrm{S}$ and to bridge the fields so that they might be mutually reinforcing. It is our hope that interested readers can profit from the insights provided by the studies reported in this survey, for instance, in understanding the factors that guide $\mathrm{C}-\mathrm{S}$ outcomes or in making use of existing annotation schema across multilingual contexts.

\section{Competing models of $\mathrm{C}-\mathrm{S}$}

For linguists, the specific ways in which languages are switched matters. The use of a single Spanish word in an English tweet (ex. 2) is not as syntactically complicated as the integration in ex. 1. In fact, it may not signal multilingualism at all, simply borrowing. Many words, particularly anglicisms, circulate globally: marketing, feedback, gay.

2. This is a good baile!

'This is a good dance party!' (Solorio and Liu, 2008)

To produce example (2), the speaker needs to know only one Spanish word. But, to produce example (1), the speaker has to know what word order and case marker to use, and which languages they should be drawn from. NLP scholars are not always concerned with the difference between examples (1) and (2) so that, with some exceptions (Bhat et al., 2016), grammatical work in NLP tends to rely heavily on the notion of a matrix language model advanced by Joshi (1982) and later adapted by Myers-Scotton (1997) as the Matrix Language Frame (MLF) model. The MLF holds that one language provides the grammatical frame into which 
words or phrases from another are embedded and its scope of application is a clause. Thus, it would not apply to the alternational English-Afrikaans $\mathrm{C}-\mathrm{S}$ in example (3) as each clause is in a separate language (Dulm, 2007).

\section{I love Horlicks maar hierś niks 'I love Horlicks but there's nothing there'}

Although it dominates computational approaches to C-S, the MLF is contested on empirical and theoretical grounds. The consistent identification of a matrix language is not always possible, the criteria for defining it are ambiguous, and its scope is limited (Meakins, 2012; Bhat et al., 2016; Adamou, 2016; MacSwan, 2000; Auer and Muhamedova, 2005). Bullock et al. (2018) computationally show that different ways of determining the matrix language only reliably converge over sentences with simple insertions as in example (2).

For many linguists, the MLF is not the only way, or even an adequate way, to theorize C-S. The Equivalence Constraint (Poplack, 1980) captures the fact that $\mathrm{C}-\mathrm{S}$ tends to occur at points where the linear structures of the contributing languages coincide, as when the languages involved share word order. Other syntactic theories are built on the differences between lexical and functional elements, including the Government Constraint (DiSciullo et al., 1986) and the Functional Head Constraint (Belazi et al., 1994). Incorporating the latter in NLP experiments has been shown to improve the accuracy of computational and speech models ( $\mathrm{Li}$ and Fung, 2014; Bhat et al., 2016). Functional elements include negative particles and auxiliaries, which are respectively classified as Adverbs and Verbs (lexical classes), in some NLP tag sets (AlGhamdi et al., 2016). This means that NLP experiments often use annotations that are too coarse to be linguistically informative with regard to C-S. Constraint-free theories (Mahootian and Santorini, 1996; MacSwan, 2000) hold that nothing restricts switching apart from the grammatical requirements of the contributing languages. Testing such theories in NLP experiments would require syntactically parsed corpora that are rare for mixed language data (Partanen et al., 2018). In sum, working together, theoretical and computational linguists could create better tools for processing C-S than those currently available.

\section{Why do speakers code-switch?}

In addition to focusing on the linguistic aspects and constraints on C-S, linguists are also interested in the social and cognitive motivations for switching across languages. What a (multilingual) speaker is trying to achieve by switching languages can affect its structural outcome. Linguists recognize that pragmatic, interactional, and socio-indexical functions may condition C-S patterns. For instance, Myslín and Levy (2015) demonstrate that Czech-English speakers switch to English for highinformation content words in prominent prosodic positions when speaking Czech. Other uses of C-S with structural traces include signalling an in-group identity through backflagging (Muysken, 1995) or emblematic tag-switching (Poplack, 1980). These are words or phrases that are used at the edge of clauses (e.g., Spanish ojalá or English so). Other functions, among these, quoting a speaker, getting the attention of an interlocutor, or reiterating an utterance to soften or intensify a message will also be indicated via $\mathrm{C}-\mathrm{S}$ in predictable linguistic constructions, such as with verbs of 'saying', vocative expressions, and sequential translation equivalents (Gumperz, 1982; Zentella, 1997).

According to Clyne (1991), there are eight factors (e.g. topic, type of interaction, interlocutors, role relationship, communication channel) that can influence C-S choices. Lavric (2007) explains C$\mathrm{S}$ choices in line with politeness theory, focusing on prestige and face-saving moves in multilingual conversations. Heller (1992) takes a macro-social view, arguing that French-English C-S in Quebec may signal a political choice among both dominant and subordinate groups.

Gardner-Chloros and Edwards (2004) suggest that social factors influence language choice, with different generations of speakers from the same community exhibiting very different C-S patterns. Similarly Sebba (1998) argues that as speakers cognitively construct equivalence between morphemes, words, and phrases across their languages, communities of the same languages may do this differently. Evidence from computational studies suggests that C-S is speaker-dependent (Vu et al., 2013). Gender and identity also play a role for C-S practices in English and Greek Cypriot community in London (Finnis, 2014). From a computational perspective, Papalexakis et al. (2014) investigated the factors that influence C-S choices (Turkish-Dutch) in computer mediated interaction and how to predict them 
automatically.

\section{Code-switching, Borrowing, Transfer, Loan Translation}

While C-S implies active alternation between grammatical systems, borrowing does not. It is difficult to know if a lone word insertion (e.g. example (2)) constitutes a borrowing or a C-S without considering how the items are integrated into the grammar of the receiving language (Poplack et al., 1988). When such analyses are done, most lone-item insertions are analyzable as one-time borrowings, called nonce borrowings (Sankoff et al., 1990). Similarly, what looks like complex C-S may not be perceived as switching at all. Auer (1999) distinguishes a continuum of mixing types: prototypical C-S is pragmatic and intentional, Language Mixing serves no pragmatic purpose, and Mixed Languages are the single code of a community. These can look structurally identical, but the latter can be modeled as a single language (e.g. languages like Michif Cree (Bakker, 1997) or Gurinji Kriol (Meakins, 2012)) rather than the intertwining of two. Bilaniuk (2004) describes the Surzhyk spoken by urban Russian-Ukrainian bilinguals (in Ukraine) as 'between C-S and Mixed Language' since speakers are highly bilingual and the direction of switching is indeterminate.

Loan translation and transfer involve the words from only one language but the semantics and grammatical constructions from the other. In example 4, the Turkish verb yapmak, ' to do', takes on the Dutch meaning of doen in Turkish spoken in the Netherlands (Doğruöz and Backus, 2009).

4. İlkokul-u İstanbul-da yap-tı-m. primary.school-ACC İstanbul-LOC do-past1 sg.

'I finished primary school in Istanbul.'

In transfer, grammatical constructions can be borrowed from one language to another without the words being borrowed. Treffers-Daller (2012) demonstrates the transfer of verb particles from Germanic languages into French. In Brussels French (Belgium), the construction chercher après 'look after' (for 'look for') is a translation of the Dutch equivalent and, in Ontario French (Canada), chercher pour is the translation equivalent of English 'look for'. In reference French (France), there is normally no particle following the verb. The degree to which linguistic features like loan translation and transfer can be found alongside C-S is unknown.

\section{C-S across Languages: European Context}

The contexts in which people acquire and use multiple languages in Europe are diverse. Some acquire their languages simultaneously from birth, while others acquire them sequentially, either naturally or via explicit instruction. Multilingualism is the norm in many zones where local residents may speak different languages to accommodate their interlocutors. Speakers who use local dialects or minoritized varieties may also be engaged in C-S when switching between their variety and a dominant one (Mills and Washington, 2015; Blom and Gumperz, 1972).

$\mathrm{C}-\mathrm{S}$ in bilingual language acquisition of children has been studied across language contact contexts in Europe. In Germany, Herkenrath (2012) and Pfaff (1999) focused on Turkish-German C-S and Meisel (1994) on German-French C-S of bilingual children. From a comparative perspective, Poeste et al. (2019) analyzed C-S among bilingual, trilingual, and multilingual children growing up in Spain and Germany. In the Netherlands, Bosma and Blom (2019) focused on C-S among bilingual Frisian-Dutch children. In addition to analyzing C-S in children's speech, Juan-Garau and PerezVidal (2001) and Lanza (1998) investigated C-S in the interaction patterns between bilingual children and their parents (i.e. Spanish-Catalan and English-Norwegian respectively).

Within an educational setting, Kleeman (2012) observed C-S among bilingual (North SamiNorwegian) kindergarten children in the North of Norway. Similarly, Jørgensen (1998) and Cromdal (2004) report the use of C-S for resolving disputes among bilingual (Turkish-Danish) children in Denmark and multilingual (Swedish-English and/or a Non-Scandinavian Language) children in Sweden respectively.

C-S does not only take place between standard languages but between minority languages and dialects as well. For example, Themistocleous (2013) studied C-S between Greek and Cypriot Greek and Deuchar (2006) focused on the C-S between Welsh and English in the UK. Berruto (2005) reports cases of language mixing between standard Italian and Italoromance dialects in Italy. In the 
Balkans, Kyuchukov (2006) analyzed C-S between Turkish-Bulgarian and Romani in Bulgaria. C-S between dialects and/or standard vs. minority languages in computer mediated interaction was analyzed by Siebenhaar (2006) among Swiss-German dialects and by Robert-Tissot and Morel (2017) through SMS corpora collected across Germanic (i.e. English and German) and Romance languages (French, Spanish, Italian) in Switzerland.

C-S is commonly observable across immigrant contexts in Europe. In the UK, Georgakopoulou and Finnis (2009) described the C-S patterns between English and Cypriot Greek while Issa (2006) focused on the C-S between English and Cypriot Turkish communities in London. Wei and Milroy (1995) analyzed the C-S between English and Chinese from a conversational analysis point of view based on the interactions of bilingual (ChineseEnglish) families in Northeastern England. In addition, Ożańska-Ponikwia (2016) investigated the Polish-English C-S in the UK as well. C-S among immigrant community members have also been widely studied in Germany (e.g. TurkishGerman C-S by Keim (2008) and Çetinoğlu (2017), Russian-German C-S by Khakimov (2016)). In the Netherlands, C-S studies include Turkish-Dutch C-S by Backus (2010) and Dutch-Morroccan C-S by Nortier (1990). In Belgium, Meeuws and Blommaert (1998) studied the French-Lingala-Swahili C-S among immigrants of Zaire and Treffers-Daller (1994) studied French-Dutch C-S in Brussels. In Spain, Jieanu (2013) describes the RomanianSpanish C-S among the Romanian immigrants. In addition to the C-S analyses within spoken interactions of immigrant communities across Europe, there are also studies about C-S within computer mediated communication as well. These studies include Greek-German C-S by Androutsopoulos (2015) in Germany, Turkish-Dutch C-S by Papalexakis et al. (2014), Papalexakis and Doğruöz (2015) and a comparison of Turkish-Dutch and MoroccanDutch C-S by Dorleijn and Nortier (2009) in the Netherlands. Similarly, Marley (2011) compared French-Arabic C-S within computer mediated interaction across Moroccan communities in France and the UK.

In addition to daily communication, some linguists are also interested in the C-S observed in historical documents. While Swain (2002) explored Latin-Greek C-S by Cicero (Roman Statesman), Dunkel (2000) analyzed C-S in his communication with Atticus (Roman philosopher who studied in Athens) in the Roman Empire. Argenter (2001) reports cases of language mixing within the Catalan Jewish community (in Spain) in the 14th and 15th centuries and Rothman (2011) highlights the C-S between Italian, Slavic and Turkish in the historical documents about Ottoman-Venetian relations. In Switzerland, Volk and Clematide (2014) worked on detecting and annotating C-S patterns in diachronic and multilingual (English, French, German, Italian, Romansh and Swiss German) Alpine Heritage corpus.

Within the media context, Martin (1998) investigated English C-S in written French advertising, and Onysko (2007) investigated the English C-S in German written media through corpus analyses. Zhiganova (2016) indicates that German speakers perceive C-S into English for advertising purposes with both positive and negative consequences.

Similar to humans, institutions and/or organizations could also have multilingual communication with their members and/or audience. For example, Wodak et al. (2012) analyzed the C-S and language choice at the institutional level for European Union institutions.

\section{C-S across Languages: Indian Context}

According to the 2011 Census (Chandramouli, 2011), 26\% of the population of India is bilingual, while $7 \%$ is trilingual. There are 121 major languages and 1599 other languages in India, out of which 22 (Assamese, Bangla, Bodo, Dogri, Gujarati, Hindi, Kashmiri, Kannada, Konkani, Maithili, Malayalam, Manipuri, Marathi, Nepali, Oriya, Punjabi, Tamil, Telugu, Sanskrit, Santali, Sindhi, Urdu) are scheduled languages with an official recognition (almost $97 \%$ of the population speaks one of the scheduled languages). Most of the population (93\%) speak languages from the Indo-Aryan (Hindi, Bengali, Marathi, Urdu, Gujarati, Punjabi, Kashmiri, Rajasthani, Sindhi, Assamese, Maithili, Odia) and Dravidian (Kannada, Malayalam, Telugu, Tamil) language families. The census excludes languages with a population lower than 10,000 speakers. Given this, it is probably difficult to find monolingual speakers in India considering the linguistic diversity and wide-spread multilingualism.

Kachru (1978) provides one of the early studies on the types and functions of C-S in India with a historical understanding of the multilingual context. 
In addition to the mutual influences and convergence of Indo-Aryan and Dravidian languages internally, he mentions Persian and English as outside influences on Indian languages. Similarly, Sridhar (1978) provides an excellent comparative overview about the functions of C-S in Kannada in relation to the Perso-Arabic vs. English influences. Kumar (1986) gives examples about the formal (e.g. within NPs, PPs, VPs) and functional (i.e. social and stylistic) aspects of Hindi-English C-S from a theoretical point of view. More recently, Doley (2013) explains how fish mongers in a local fish market in Assam adjust and switch between Assamese, English and local languages strategically to sell their products to multilingual clientele. Another observation about C-S in daily life comes from Boro (2020) who provides examples of English, Assamese and Bodo (another language spoken in the Assam region) C-S and borrowings. In addition to English, Portuguese was also in contact with the local languages as a result colonization in South India. For example, Kapp (1997) explains the Portuguese influence through borrowings in Dravidian languages (i.e. Kannada and Telugu) spoken in India.

Instead of automatic data collection and methods of analyses, the C-S examples for the abovementioned studies are (probably) encountered and collected by the authors themselves in daily life interactions over a period of time with limited means. Nowadays, these small sets of data would be regarded as insignificant in computational areas of research. However, ignoring these studies and data could have serious consequences since crucial information about the social and cultural dynamics in a multilingual setting would also be lost. For example, Nadkarni (1975) proves this point by explaining how social factors influence the C-S between Saraswat Brahmin dialect of Konkani (Indo-Aryan language) and Kannada (Dravidian language) in the South of India. Both languages have been in contact with each other for over four hundred years. Saraswat Brahmins are fluent in both Konkani and Kannada but they do not speak Konkani with Kannada speakers and they also do not C-S between Konkani and Kannada. Nadkarni (1975) attributes this preference to the high prestige associated with Konkani within the given social context. Since Kannada (perceived as less prestigious) is widely spoken in that region, Konkani speakers learn and speak Kannada for functional purposes in daily life which does not involve C-S. However, it is not common for Kannada speakers to learn and speak Konkani (Nadkarni, 1975).

$\mathrm{C}-\mathrm{S}$ in India has been investigated through written media, advertising and film industry as well. Si (2011) analyzed Hindi-English C-S in the scripts of seven Bollywood movies which were filmed between 1982 and 2004. Her results indicate a change of direction C-S over the years. More specifically, Hindi was the dominant language with occasional switches to English for the early productions but English became the dominant language especially for younger generations in the later productions. A similar trend has been observed for Bengali movie scripts as well. Through analyzing movie scripts (between 1970s and 2010s), Chatterjee (2016) finds a drastic increase in the use of bilingual verbs (e.g. renovate koreche "renovation do") over time and attributes this rise to the increasing popularity of English in Indian society. Within the immigrant context, Gardner-Chloros and Charles (2007) focused on the types and functions of C-S between Hindi and English across the TV programs (e.g. highly scripted vs. loosely scripted programs) of a British/Asian cable channel in the UK. Although they have come across C-S in a variety of TV shows, the least amount of C-S was encountered in the news broadcasts (i.e. highly scripted). In general, they have encountered less C-S on TV broadcasts in comparison to the natural speech and attribute this factor to the consciousness of TV personalities about pure language use (instead of C-S). Similarly, Zipp (2017) analyzed Gujarati-English C-S within a radio show targeting British South Asians living in the US and concluded that C-S was part of identity construction among youngsters (group identity). Pratapa and Choudhury (2017) perform a quantitative study of 18 recent Bollywood (Hindi) movies and find that C-S is used for establishing identity, social dynamics between characters and the socio-cultural context of the movie.

From an advertising point of view, Kathpalia and Wee Ong (2015) analyzed C-S in Hinglish (i.e. Hindi, English, Urdu, Sanskrit according to their definition) billboards about the Amul brand in India. After compiling the advertisements on billboards (1191), they classified the structures and functions of C-S. Their results indicate more intrasentential C-S than intersentential ones on the billboards. In terms of function, the advertisers used C-S to indicate figures of speech 
(e.g. puns, associations, contradictory associations, word-creation and repetitions) to attract the attention of the target group.

Mohanty (2006) provides an extended overview of the multilingual education system in India exploring the types and quality of schools across a wide spectrum. In general, high-cost English Medium (EM) education is valued by upper-class and affluent families. Although low-cost EM education is also available for lower income families, he questions its impact in comparison to education in the local languages. Sridhar (2002) explains that $\mathrm{C}-\mathrm{S}$ is commonly practiced among students in schools across India. In addition, she finds it unrealistic to ask the students to separate the two languages harshly. In immigrant contexts, Martin et al. (2006) investigates how Gujarati-English C-S is used among the South Asian students in educational settings in the UK. Another analysis reveals a shift from Bengali toward English among the younger generations of the immigrant Bengali community in the UK (Al-Azami, 2006). In terms of the C-S patterns, first generation immigrants integrate English words while speaking Bengali whereas English dominates the conversations of younger generations with occasional switches to Bengali. There are also studies about Bengali-English C-S in the UK school settings (Pagett, 2006) and Bangladesh (Obaidullah, 2016) as well. However, a systematic comparison between Bengali-English C-S in India, Bangladesh and immigrant settings are lacking.

In their study about aphasic patients, Shyamala Chengappa and Bhat (2004) report increased frequency of C-S between Malayalam and English for aphasic patients in comparison to the control group. However, there were less differences between the groups in terms of functions of C-S. Deepa and Shyamala (2019) find that amount and types of C-S could be used to differentiate between healthy and mild dementia patients who are bilingual in Kannada and English. Although both studies are carried out with limited subjects, they offer insights about the use of $\mathrm{C}-\mathrm{S}$ in health settings as well.

\section{Computational Approaches to C-S}

There has been significant interest in building language technologies for code-switched languages over the last few years, spanning a diverse range of tasks such as Language Identification, Part of Speech Tagging, Sentiment Analysis and Au- tomatic Speech Recognition. In the European language context, work has mainly focused on Turkish-Dutch, Frisian-Dutch, Turkish-German and Ukranian-Russian with some initial attempts being made in parsing Russian-Komi text. In the Indian language context, Hindi-English is the most widely studied language pair for computational processing, with some recent work on Telugu-English, Tamil-English, Bengali-English and Gujarati-English. Sitaram et al. (2019) provide a comprehensive survey of research in computational processing of C-S text and speech and Jose et al. (2020) present a list of datasets available for $\mathrm{C}-\mathrm{S}$ research. However, despite significant efforts, language technologies are not yet capable of processing C-S as seamlessly as monolingual data. We identify three main limitations of the current state of computational processing of C-S: data, evaluation and user-facing applications.

\subsection{Data}

The use of Deep Neural Networks, which require large amounts of labeled and unlabeled training data have become the de facto standard for building speech and NLP systems. Since C-S languages tend to be low resourced, building Deep Learningbased models is challenging due to the lack of large C-S datasets. Massive multilingual Language Models (LMs) such as multilingual BERT (Devlin et al., 2019) and XLM-R (Conneau et al., 2020) have shown promise in enabling the coverage of lowresource languages without any labeled data by using the zero-shot framework. These LMs are typically trained in two phases: a "pre-training" phase, in which unlabeled data from one or multiple languages may be used and a "fine-tuning" phase, in which task-specific labeled data is used to build a system capable of solving the task.

Since multilingual LMs are trained on multiple languages at the same time, it has been suggested that these models may be capable of processing C-S text (Johnson et al., 2017), with promising results initially reported on POS tagging (Pires et al., 2019). Khanuja et al. (2020) found that multilingual BERT outperforms older task-specific models on C-S tasks, however, the performance on C-S is much worse than the performance on the same tasks in a monolingual setting. Further, these LMs are either trained primarily on monolingual datasets such as Wikipedia in the case of mBERT, or Com- 
mon Crawl ${ }^{1}$ in the case of XLM-R. So, they are either not exposed to C-S data at all during training, or they miss out on several language pairs, types and functions of $\mathrm{C}-\mathrm{S}$ that are encountered in daily life but not available on the web.

Since massive multilingual LMs are now replacing traditional models across many NLP applications, it is crucial to consider how they can be trained on C-S data, or made to work for C-S by incorporating other sources of knowledge.

\subsection{Evaluation}

Much of speech and NLP research is now driven by standard benchmarks that evaluate models across multiple tasks and languages. Due to the shortage of standardized datasets for C-S, until recently, the evaluation of C-S models was performed over individual tasks and language pairs. Khanuja et al. (2020) and Aguilar et al. (2020) proposed the first evaluation benchmarks for C-S that span multiple tasks in multiple language pairs. The GLUECoS benchmark (Khanuja et al., 2020) consists of the following C-S tasks in Spanish-English and Hindi-English: Language Identification (LID), Part of Speech (POS) tagging, Named Entity Recognition (NER), Sentiment Analysis, Question Answering and Natural Language Inference (NLI). The LINCE benchmark (Aguilar et al., 2020) covers Language Identification, Named Entity Recognition, Part-of-Speech Tagging, and Sentiment Analysis in four language pairs: Spanish-English, Nepali-English, Hindi-English, and Modern Standard Arabic-Egyptian Arabic.

Although these benchmarks are important starting points for $\mathrm{C}-\mathrm{S}$, it is clear that they do not represent the entire spectrum of C-S, both from the point of view of potential applications and language pairs. Further, it is important to note that while state-ofthe-art models perform well on tasks such as LID, POS tagging and NER, they are only slightly better than chance when it comes to harder tasks like NLI, showing that current models are not capable of processing C-S language. Moreover, many of the C-S tasks in the benchmarks above consist of annotated tweets, which only represent a certain type of C-S. Due to these limitations, we currently do not have an accurate picture of how well models are able to handle C-S.

\footnotetext{
${ }^{1}$ http://www.commoncrawl.org
}

\subsection{User-facing applications}

Although speech and NLP models for C-S have been built for various applications, a major limitation of the work done so far in computational processing of C-S is the lack of end-to-end userfacing applications that interact directly with users in multilingual communities. For example, there is no widely-used spoken dialogue system that can understand as well as produce code-switched speech, although some voice assistants may recognize and produce $\mathrm{C}-\mathrm{S}$ in limited scenarios in some locales. Although computational implementations of grammatical models of C-S exist (Bhat et al., 2016), they do not necessarily generate natural C-S utterances that a bilingual speaker would produce (Pratapa et al., 2018). Most crucially, current computational approaches to C-S language technologies do not usually take into account the linguistic and social factors that influence why and when speakers/users choose to code-switch.

Bawa et al. (2020) conducted a Wizard-of-Oz study using a Hindi-English chatbot and found that not only did bilingual users prefer chatbots that could code-switch, they also showed a preference towards bots that mimicked their own C-S patterns. Rudra et al. (2016) report a study on 430k tweets from Hindi-English bilingual users and find that Hindi is preferred for the expression of negative sentiment. In a follow-up study, Agarwal et al. (2017) find that Hindi is the preferred language for swearing in Hindi-English C-S tweets, and swearing may be a motivating factor for users to switch to Hindi. The study also finds a gender difference, with women preferring to swear in English more often than Hindi. Such studies indicate that multilingual chatbots and intelligent agents need to be able to adapt to users' linguistic styles, while also being capable of determining when and how to code-switch.

Due to the paucity of user-facing systems and standard benchmarks covering only a handful of simpler NLP tasks, it is likely that we overestimate how well computational models are able to handle C-S. In sum, language technologies for C-S seem to be constrained by the lack of availability of diverse C-S training data, evaluation benchmarks and the absence of user-facing applications. They need to go beyond pattern recognition and grammatical constraints of C-S in order to process and produce C-S the way humans do. Hence, it is important for the CL community to be aware of the vast litera- 
ture around C-S in linguistics, particularly as we proceed to solving more challenging tasks.

\section{Conclusion}

The goal of this paper was to inform computational linguists and language technologists about the linguistic and social aspects C-S studies focusing on the European and Indian multilingual contexts. There are some similarities (e.g. themes for linguistic research in C-S) but also differences between the two contexts in terms of the social, cultural and historical characteristics. For example, $\mathrm{C}-\mathrm{S}$ in immigrant communities has been a common theme for both multilingual contexts. In Europe, $\mathrm{C}-\mathrm{S}$ has been widely studied within the immigrant communities who have come through labor immigration in the 1960s. However, there is a need for more research about the C-S in immigrant languages with a more recent history as well as minority languages and regional dialects. Analyzing C-S in the immigration context is even more complex for Indian languages. There are hardly any systematic linguistic comparisons between the $\mathrm{C}-\mathrm{S}$ within the same language pairs in India and immigrant contexts (e.g. C-S between Hindi-English in India vs. Hindi-English in the US/UK). There is also a need for more research about C-S between less known language pairs in India. However, some of these languages are not even officially listed (e.g. in census results) since they have less than 10,000 speakers. In these cases, collecting and analyzing the multilingual and C-S data becomes more difficult.

A common flaw that is shared both by linguistics and computational areas of research is to focus only on the positive evidence and assume that C-S will occur in all multilingual contexts. However, there is also a need for negative evidence to falsify this assumption. As illustrated through an example from Konkani-Kannada language contact in India (see section 6), bilingual speakers may prefer not to C-S due to historical, social and cultural factors operating in that setting. Therefore, developing an automatic C-S system for a random pair of languages without an in-depth and systematic analysis of linguistic and social aspects of C-S in a particular context would not be very useful for the targeted users and/or language technologists.

To date, the literature focusing on the social and linguistic aspects of C-S is less visible for CL researchers. This lack of visibility leads to misunder- standings and/or incomplete citations of earlier research which would have saved time and resources for CL research if resolved. One of the reasons is perhaps the differences in publishing traditions between humanities and computational areas of research. Conference and workshop proceedings are commonly accepted means of publication in computational linguistics. Whereas, journal publications, books and/or chapters are the publication forms in humanities. However, guidelines about how to cite publications in humanities are often missing in computational venues. There are also differences in terms of length, review cycles and open access policies between the two fields which may influence the visibility of research output for each other. It is perhaps useful to remember that science advances by standing on the shoulders of giants (i.e. building upon earlier research). With our contribution to the conference, we hope to connect the two fields and start a scientific dialogue to enhance the advancement in both fields.

\section{References}

Evangelia Adamou. 2016. A corpus-driven approach to language contact: Endangered languages in a comparative perspective, volume 12 . Walter de Gruyter GmbH \& Co KG.

Prabhat Agarwal, Ashish Sharma, Jeenu Grover, Mayank Sikka, Koustav Rudra, and Monojit Choudhury. 2017. I may talk in English but gaali toh Hindi mein hi denge: A study of EnglishHindi code-switching and swearing pattern on social networks. In 2017 9th International Conference on Communication Systems and Networks (COMSNETS), pages 554-557. IEEE.

Gustavo Aguilar, Sudipta Kar, and Thamar Solorio. 2020. Lince: A centralized benchmark for linguistic code-switching evaluation. In Proceedings of The 12th Language Resources and Evaluation Conference, pages 1803-1813.

Salman Al-Azami. 2006. Linguistic manipulations in the Bengali language by the Bangladeshis in Manchester. South Asian Cultural Studies, 1(1):53-59.

Fahad AlGhamdi, Giovanni Molina, Mona Diab, Thamar Solorio, Abdelati Hawwari, Victor Soto, and Julia Hirschberg. 2016. Part of speech tagging for code switched data. Proceedings of the Second Workshop on Computational Approaches to CodeSwitching.

Jannis Androutsopoulos. 2015. Networked multilingualism: Some language practices on facebook and their implications. International Journal of Bilingualism, 19:185-205. 
Joan A. Argenter. 2001. Code-switching and dialogism: Verbal practices in among Catalan Jews in the middle ages. Language in Society, 30:377-402.

Peter Auer. 1999. From codeswitching via language mixing to fused lects: Toward a dynamic typology of bilingual speech. International journal of bilingualism, 3(4):309-332.

Peter Auer and Raihan Muhamedova. 2005. Embedded language' and 'matrix language'in insertional language mixing: Some problematic cases. Rivista di linguistica, 17(1):35-54.

Ad Backus. 2010. The role of codeswitching, loan translation and interference in the emergence of an immigrant variety of Turkish. Working Papers in Corpus-based Linguistics and Language Education, $5: 225-241$

Peter Bakker. 1997. A language of our own: The genesis of Michif, the mixed Cree-French language of the Canadian Métis. Oxford University Press.

Anshul Bawa, Pranav Khadpe, Pratik Joshi, Kalika Bali, and Monojit Choudhury. 2020. Do multilingual users prefer chat-bots that code-mix? let's nudge and find out! Proceedings of the ACM on Human-Computer Interaction, 4(CSCW1):1-23.

Hedi M. Belazi, Edward J Rubin, and Almeida Jacqueline Toribio. 1994. Code switching and x-bar theory: The functional head constraint. Linguistic inquiry, pages 221-237.

Gaetano Berruto. 2005. Dialect/standard convergence, mixing, and models of language contact: The case of Italy. In Peter Auer, Franz Hinskens, and Paul Kerswill, editors, Dialect Change: Convergence and Divergence in European Languages, pages 81-95. Cambridge University Press, Cambridge.

Gayatri Bhat, Monojit Choudhury, and Kalika Bali. 2016. Grammatical constraints on intra-sentential code-switching: From theories to working models. arXiv preprint arXiv: 1612.04538.

Laada Bilaniuk. 2004. A typology of Surzhyk: Mixed Ukrainian-Russian language. International Journal of Bilingualism, 8(4):409-425.

Jan-Petter Blom and John Gumperz. 1972. Social meaning in linguistic structures: Code switching in Northern Norway. In John Gumperz and Del Hymes, editors, Directions in Sociolinguistics: The Ethnography of Communication. Holt, Rinehart and Winston, New York.

Jupitara Boro. 2020. Borrowing or code-switching: Some observations in Bodo. Language in India, 20(7):87-94.

Evelyn Bosma and Elma Blom. 2019. A codeswitching asymmetry in bilingual children: Codeswitching from Dutch to Frisian requires more cognitive control than code-switching from Frisian to Dutch. International Journal of Bilingualism, 23:1431-1447.

Barbara Bullock, Wally Guzmán, Jacqueline Serigos, Vivek Sharath, and Almeida Jacqueline Toribio. 2018. Predicting the presence of a matrix language in code-switching. In Proceedings of the third workshop on computational approaches to linguistic code-switching, pages 68-75.

Özlem Çetinoğlu. 2017. A code-switching corpus of Turkish-German conversations. In Proceedings of the 11th Linguistic Annotation Workshop, pages 34 40, Valencia, Spain. Association for Computational Linguistics.

C Chandramouli. 2011. Census of India 2011. Provisional Population Totals, Office of Registrar General and Census Commissioner, New Delhi: Government of India, pages 409-413.

Tridha Chatterjee. 2016. Structural changes in BengaliEnglish bilingual verbs through the exploration of Bengali films. Languages, 1(5):2-17.

Michael Clyne. 1991. Community Languages: The Australian experience. Cambridge University Press, Cambridge.

Alexis Conneau, Kartikay Khandelwal, Naman Goyal, Vishrav Chaudhary, Guillaume Wenzek, Francisco Guzmán, Édouard Grave, Myle Ott, Luke Zettlemoyer, and Veselin Stoyanov. 2020. Unsupervised cross-lingual representation learning at scale. In Proceedings of the 58th Annual Meeting of the Association for Computational Linguistics, pages 8440 8451.

Jakob Cromdal. 2004. Building bilingual oppositions: Code-switching in children's disputes. Language in Society, 33:33-58.

M.S. Deepa and K.C. Shyamala. 2019. Analysis of verb expressions in the conversational speech of Kannada-English speaking bilingual persons with mild dementia. Studies in Linguistics and Literature, 3:182-194

Margaret Deuchar. 2006. Welsh-English codeswitching and the Matrix Language Frame model. Lingua, 116:1986-2011.

Jacob Devlin, Ming-Wei Chang, Kenton Lee, and Kristina Toutanova. 2019. Bert: Pre-training of deep bidirectional transformers for language understanding. In Proceedings of the 2019 Conference of the North American Chapter of the Association for Computational Linguistics: Human Language Technologies, Volume 1 (Long and Short Papers), pages 4171-4186.

Anne-Marie DiSciullo, Pieter Muysken, and Rajendra Singh. 1986. Code-mixing and government. Journal of Linguistics, 22(1):1-24. 
Rajeev K. Doley. 2013. Code-switching at the market place: The trader's tool. Asian Journal of Social Sciences and Humanities, 2(2):483-489.

Margreet Dorleijn and Jacomine Nortier. 2009 Codeswitching and the internet. In Barbara Bullock and Almeida Jacqueline Toribio, editors, The Cambridge Handbook of Linguistic Code-switching, pages 127-141. Cambridge University Press, Cambridge.

A. Seza Doğruöz and Ad Backus. 2009. Innovative constructions in Dutch Turkish: An assessment of on-going contact-induced change. Bilingualism: Language and Cognition, 12(1):41-63.

Ondene Van Dulm. 2007. The grammar of EnglishAfrikaans code switching: A feature checking account. Ph.D. thesis, Radboud Universiteit, Nijmegen.

George E. Dunkel. 2000. Remarks on code-switching in Cicero's letters to Atticus. Museum Helveticum, 57:122-129.

Katerina A. Finnis. 2014. Variation within a GreekCypriot community of practice in London: Codeswitching, gender, and identity. Language in Society, 43:287-310.

Penelope Gardner-Chloros and Reeva Charles. 2007. Subiko welcome (welcome to everyone) HindiEnglish code-switching in the British-Asian media. BISAL, 2:89-122.

Penelope Gardner-Chloros and Malcolm Edwards. 2004. Assumptions behind grammatical approaches to code-switching: When the blueprint is a red herring. Transactions of the Philological Society, 102:103-129.

Alexandra Georgakopoulou and Katerina Finnis. 2009. Code-switching in site for fantasizing identities a case study of conventional uses of London Greek Cypriot. Pragmatics, 19:467-488.

John J. Gumperz. 1982. Discourse strategies, volume 1. Cambridge University Press.

Monica Heller. 1992. The politics of codeswitching and language choice. In Carol Eastman, editor, Codeswitching, pages 108-118. Multilingual Matters, Clevendon.

Annette Herkenrath. 2012. Receptive multilingualism in an immigrant constellation: Examples from Turkish-German children's language. International Journal of Bilingualism, 16:287-314.

Tözün Issa. 2006. An ethnographic case study of code switching and language choice: The uses of Cypriot Turkish in London. International Journal of Sociology of Language, 181:1-9.

Ioana Jieanu. 2013. Aspects of code-switching concerning Spanish-Romanian virtual communication. Journal for Foreign Languages, 5:21-33.
Melvin Johnson, Mike Schuster, Quoc V Le, Maxim Krikun, Yonghui Wu, Zhifeng Chen, Nikhil Thorat, Fernanda Viégas, Martin Wattenberg, Greg Corrado, et al. 2017. Google's multilingual neural machine translation system: Enabling zero-shot translation. Transactions of the Association for Computational Linguistics, 5:339-351.

Navya Jose, Bharathi Raja Chakravarthi, Shardul Suryawanshi, Elizabeth Sherly, and John P McCrae. 2020. A survey of current datasets for codeswitching research. In 2020 6th International Conference on Advanced Computing and Communication Systems (ICACCS), pages 136-141. IEEE.

Aravind Joshi. 1982. Processing of sentences with intra-sentential code-switching. In Coling 1982: Proceedings of the Ninth International Conference on Computational Linguistics.

Maria Juan-Garau and Carmen Perez-Vidal. 2001. Mixing and pragmatic parental strategies in early bilingual acquisition. Journal of Child Language, 28:59-86.

Normann J. Jørgensen. 1998. Children's acquisition of code-switching for power-wielding. In Peter Auer, editor, Code-switching in conversation: Language, interaction and identity, pages 97-113. Routledge, London.

Braj B. Kachru. 1978. Toward structuring codemixing: An Indian perspective. International Journal of the Sociology of Language, 1978(16):27-46.

Dieter B. Kapp. 1997. Dravidian borrowings from Portuegese (Kannada and Telugu). In Charles J. Borges and Helmut Feldmann, editors, Goa and Portugal: Their Cultural Links, pages 280-301. Ashok Kumar Mottar, New Delhi.

Birsel Karakoç and Annette Herkenrath. 2019. Receptive multilingualism in an immigrant constellation: Examples from Turkish-German children's language. Turkic Languages, 23:81-122.

Sujata S. Kathpalia and Kenneth Keng Wee Ong. 2015. The use of code-mixing in Indian billboard advertising. World Englishes, 34(4):557-575.

Inken Keim. 2008. Die Türkischen Powergirls" - Lebenswelt und kommunikativer Stil einer Migrantinnengruppe in Mannheim. Narr, Tubingen.

Nikolay Khakimov. 2016. Effects of frequency and word repetition on switch placement: Evidence from Russian-German code-mixing. In Justyina Robinson and Monica Heller, editors, Cognitive Perspectives on Bilingualism, pages 91-125. De Gruyter, Berlin, Boston.

Simran Khanuja, Sandipan Dandapat, Anirudh Srinivasan, Sunayana Sitaram, and Monojit Choudhury. 2020. Gluecos: An evaluation benchmark for codeswitched nlp. In Proceedings of the 58th Annual Meeting of the Association for Computational Linguistics, pages 3575-3585. 
Carola Kleeman. 2012. Play in two languages. Language alternation and code-switching role-play in North Sámi and Norwegian. Nordlyd, 39:47-69.

Ashok Kumar. 1986. Certain aspects of the form and functions of Hindi-English code-switching. Anthropological Linguistics, 28(2):195-205.

Hristo Kyuchukov. 2006. Code-switching among Muslim Romas in Bulgaria. International Journal of Sociology of Language, 179:41-51.

Elizabeth Lanza. 1998. Raising children bilingually in Norway. International Journal of Sociology of Language, 133:73-88.

Eva Lavric. 2007. Code choice and face. Lodz Papers in Pragmatics, 3:23-35.

Ying Li and Pascale Fung. 2014. Language modeling with functional head constraint for code switching speech recognition. In Proceedings of the 2014 Conference on Empirical Methods in Natural Language Processing (EMNLP), pages 907-916.

Jeff MacSwan. 2000. The architecture of the bilingual language faculty: Evidence from codeswitching. Bilingualism: Language and Cognition, 3:3754.

Shahrzad Mahootian and Beatrice Santorini. 1996. Code switching and the complement/adjunct distinction. Linguistic Inquiry, pages 464-479.

Dawn Marley. 2011. Code-switching in websites for the Moroccan diaspora. In Foued Laroussi, editor, Code-Switching, Languages in Contact and Electronic Writings. Peter Lang, Frankfurt.

Elizabeth Martin. 1998. The use of English in written French advertising: A study of code-switching, code-mixing and borrowing in a commercial context Studies in Linguistic Sciences, 28:159-184.

Peter Martin, Arvind Bhatt, Nirmala Bhojani, and Angela Creese. 2006. Managing bilingual interaction in a Gujarati complementary school in Leicester. Language and Education, 20(1):5-22.

Felicity Meakins. 2012. Which mix - code-switching or a mixed language? Gurindji Kriol. Journal of Pidgin and Creole languages, 27(1):105-140.

Michael Meeuws and Jan Blommaert. 1998. A monolectal view of code-switching: Layered codeswitching among Zairians in Belgium. In Peter Auer, editor, Code-Switching in Conversation: Language, Interaction and Identity, pages 76-101. Routledge, New York.

Jurgen Meisel. 1994. Children: The acquisition of grammatical constraints. Studies in Second Language Acquisition, 16:413-419.
Monique T. Mills and Julie A. Washington. 2015. Managing two varieties: Code-switching in the educational context. In Lisa J.Green Jennifer Bloomquist and Sonja L. Lanehart, editors, Oxford handbook of African American language, pages 566-581. Oxford University Press New York, New York.

Ajit K. Mohanty. 2006. Multilingualism of the unequals and predicaments of education in india: Mother tongue or other tongue?: Languages in education and glocalization. In Ofelia García, Tove Skutnabb-Kangas, and María E. Torres-Guzmán, editors, Imagining Multilingual Schools: Languages in Education and Glocalization, pages 262-279. Multilingual Matters, Bristol.

Pieter Muysken. 1995. Code-switching and grammatical theory. In Lesley Milroy and Pieter Muysken, editors, One Speaker, Two Languages: Crossdisciplinary Perspectives on Code-switching, pages 177-98. Cambridge University Press, Cambridge.

Carol Myers-Scotton. 1997. Duelling languages: Grammatical structure in codeswitching. Oxford University Press.

Mark Myslín and Roger Levy. 2015. Code-switching and predictability of meaning in discourse. Language, 91(4):871-905.

Mangesh V. Nadkarni. 1975. Bilingualism and syntactic change in Konkani. Language, 51(3):672-683.

Jacomine Nortier. 1990. Dutch-Moroccan Code Switching among Moroccans in the Netherlands. Foris Publications, Dordrecht.

Md. Obaidullah. 2016. Code switching in EFL classrooms: A Bangladeshi perspective. Theory and Practice in Language Studies, 6(5):924-934.

Alexander Onysko. 2007. Anglicism in German: Borrowing, Lexical Productivity and Written Codeswitching. Walter de Gruyter, Berlin, New York.

Katarzyna Ożańska-Ponikwia. 2016. Code-switching practices among immigrant Polish L2 users of English. Theory and Practice of Second Language Acquisition, 1:87-102.

Linda Pagett. 2006. Mum and Dad prefer me to speak Bengali at home: Code switching and parallel speech in a primary school setting. Literacy, 40.

Evangelos E. Papalexakis and A. Seza Doğruöz. 2015. Understanding multilingual social networks in online immigrant communities. In WWW'15 Companion: Proceedings of the 24th International Conference on World Wide Web, pages 865-870. Association for Computational Linguistics.

Evangelos E. Papalexakis, Dong Nguyen, and A. Seza Doğruöz. 2014. Predicting code-switching in multilingual communication for immigrant communities. In Proceedings of The First Workshop on Computational Approaches to Code Switching, pages 42-50. Association for Computational Linguistics. 
Niko Partanen, KyungTae Lim, Michael Rießler, and Thierry Poibeau. 2018. Dependency parsing of code-switching data with cross-lingual feature representations. In International Workshop on Computational Linguistics for Uralic Languages, pages 1-17. ACL.

Carol W. Pfaff. 1999. Changing patterns of language mixing in a bilingual child. In Guus Extra and Ludo Verhoeven, editors, Bilingualism and Migration, page 97-121. Mouton de Gruyter, Berlin.

Telmo Pires, Eva Schlinger, and Dan Garrette. 2019. How multilingual is multilingual BERT? In Proceedings of the 57th Annual Meeting of the Association for Computational Linguistics, pages 49965001.

Meike Poeste, Natascha Müller, and Laia Arnaus Gil. 2019. Code-mixing and language dominance: Bilingual, trilingual and multilingual children compared. International Journal of Bilingualism, 16:459-491.

Shana Poplack. 1980. Sometimes I'll start a sentence in Spanish y termino en Espanol: Toward a typology of code-switching. Linguistics, 18:233-234.

Shana Poplack, David Sankoff, and Christopher Miller. 1988. The social correlates and linguistic processes of lexical borrowing and assimilation. Linguistics, 26:47-104

Adithya Pratapa, Gayatri Bhat, Monojit Choudhury, Sunayana Sitaram, Sandipan Dandapat, and Kalika Bali. 2018. Language modeling for code-mixing: The role of linguistic theory based synthetic data. In Proceedings of the 56th Annual Meeting of the Association for Computational Linguistics (Volume 1: Long Papers), pages 1543-1553.

Adithya Pratapa and Monojit Choudhury. 2017. Quantitative characterization of code switching patterns in complex multi-party conversations: A case study on Hindi movie scripts. In Proceedings of the 14th International Conference on Natural Language Processing (ICON-2017), pages 75-84.

Aurélia Robert-Tissot and Etienne Morel. 2017. The role of functional heads in code-switching evidence from Swiss text messages. Languages, 2:282-297.

E. Natalie Rothman. 2011. Conversion and convergence in the Venetian-Ottoman borderlands. Journal of Medieval and Early Modern Studies, 41:601-633.

Koustav Rudra, Shruti Rijhwani, Rafiya Begum, Kalika Bali, Monojit Choudhury, and Niloy Ganguly. 2016. Understanding language preference for expression of opinion and sentiment: What do HindiEnglish speakers do on Twitter? In Proceedings of the 2016 conference on empirical methods in natural language processing, pages 1131-1141.

David Sankoff, Shana Poplack, and Swathi Vanniarajan. 1990. The case of the nonce loan in Tamil. Language variation and change, 2(1):71-101.
Mark Sebba. 1998. A congruence approach to the syntax of codeswitching. International Journal of Bilingualism, 2(1):1-19.

Krupa Elizabeth Daniel Shyamala Chengappa and Sapna Bhat. 2004. Language mixing and switching in Malayalam-English bilingual Aphasics. Asia Pacific Disability Rehabilitation Journal, 15:68-76.

Aung Si. 2011. A diachronic investigation of Hindi-English code-switching, using Bollywood film scripts. International Journal of Bilingualism, 15(4):388-407.

Beat Siebenhaar. 2006. Code choice and codeswitching in Swiss-German Internet Relay Chat rooms. Journal of Sociolinguistics, 10:481-506.

Sunayana Sitaram, Khyathi Raghavi Chandu, Sai Krishna Rallabandi, and Alan W Black. 2019. A survey of code-switched speech and language processing. arXiv preprint arXiv:1904.00784.

Thamar Solorio and Yang Liu. 2008. Part-of-speech tagging for English-Spanish code-switched text. In Proceedings of the 2008 Conference on Empirical Methods in Natural Language Processing, pages 1051-1060.

Kamal Sridhar. 2002. Societal multilingualism, world Englishes: Their implications for TESOL. Indian Journal of Applied Linguistics, 28(2):83-100.

S. N. Sridhar. 1978. On the functions of code-mixing in Kannada. International Journal of the Sociology of Language, 1978(16):109-118.

Simon Swain. 2002. Bilingualism in Cicero? Evidence of code-switching. In James Noel Adams, Mark Janse, and Simon Swain, editors, Bilingualism in Ancient Society: Language contact and Written Text, pages 128-168. Oxford University Press, New York.

Christiana Themistocleous. 2013. Digital codeswitching between Cypriot and standard Greek: Performance and identity play online. International Journal of Bilingualism, 19:282-297.

Jeanine Treffers-Daller. 1994. Mixing two languages: French-Dutch contact in a comparative perspective. Mouton de Gruyter, Berlin.

Jeanine Treffers-Daller. 2012. Grammatical collocations and verb-particle constructions in Brussels French: A corpus-linguistic approach to transfer. International Journal of Bilingualism, 16(1):53-82.

Martin Volk and Simon Clematide. 2014. Detecting code-switching in a multilingual Alpine Heritage Corpus. Proceedings of the First Workshop on Computational Approaches to Code Switching.

Ngoc Thang Vu, Heike Adel, and Tanja Schultz. 2013. An investigation of code-switching attitude dependent language modeling. In International Conference on Statistical Language and Speech Processing, pages 297-308. Springer. 
Li Wei and Lesley Milroy. 1995. Conversational codeswitching in a Chinese community in Britain: A sequential analysis. Journal of Pragmatics, 23.

Ruth Wodak, Michał Krzyżanowski, and Bernhard Forchtner. 2012. The interplay of language ideologies and contextual cues in multilingual interactions: Language choice and code-switching in European Union institutions. Language in Society, 41:157186.

Ana Celia Zentella. 1997. Growing up bilingual : Puerto Rican children in New York. Blackwell, Malden, MA.

Anna Zhiganova. 2016. The study of the perception of code-switching to English in German advertising. Procedia: Social and Behavioral Sciences, 236:225229.

Lena Zipp. 2017. Code-switching in the media: Identity negotiations in a Gujarati diaspora radio program. International Journal of the Sociology of Language, 2017(247):33 - 48 . 